\title{
Degradation kinetics and pathways of isopropyl alcohol by microwave- assisted oxidation process
}

Quynh Thi Phuong Tran ${ }^{1}$, Yi-Hsueh Chuang ${ }^{2}$, Steve Tan ${ }^{3}$, Chi-Hsu Hsieh ${ }^{3}$, Tung-Yu Yang $^{3}$, and Hsin-hsin Tung ${ }^{1 *}$

${ }^{1}$ Graduate Institute of Environmental Engineering, National Taiwan University, No. 1, Sec. 4, Roosevelt Rd., Taipei 10617, Taiwan

${ }^{2}$ Institute of Environmental Engineering, National Yang Ming Chiao Tung University, 1001 University Road, Hsinchu 30010, Taiwan

${ }^{3}$ Topco Scientific Company Limited, No. 483, Sec. 2, Tiding Blvd, Taipei 11493, Taiwan

*Corresponding author. Email: htung@ntu.edu.tw; Telephone: +886-2-3366-4379

\section{SUPPLEMENTARY}

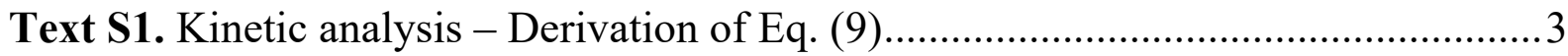

Text S2. Specific energy consumption (SEC) calculation...........................................

\section{List of Table}

Table S1. Detailed calculation of specific energy consumption in different treatment systems

Table S2. Toxicity-weighted of IPA and its metabolites in $\mathrm{MW} / \mathrm{H}_{2} \mathrm{O}_{2}$ system . .6

\section{List of Figure}

Figure S1. pH values observed during the IPA degradation experiments 7

Figure S2. Evolution of GC chromatogram of IPA and its primary degradation product (acetone) under the influence of microwave and thermal treatments 7

Figure S3. HPLC chromatogram of the generated organic acids in microwave and thermal treatment systems under the detection wavelength of $210 \mathrm{~nm}$. .8

Figure S4. Evolution of HPLC chromatogram of the generated organic acids in microwave treatment system $\left(\mathrm{MW} / \mathrm{H}_{2} \mathrm{O}_{2}\right)$ by the reaction time 


\section{List of Scheme}

Scheme S1 - Reaction pathways of the generation and degradation of pyruvic acid... 10

Scheme S2 - Degradation pathways of acetic acid............................................... 11

Scheme S3 - Degradation pathways of glycolic, glyoxylic, and oxalic acid ..............12

Scheme S4 - Degradation pathways of formic acid .......................................... 12 


\section{Text S1. Kinetic analysis - Derivation of Equation (9)}

According to Figure 2, the degradation rate of IPA can be expressed mathematically as Equation (1'):

$$
\frac{\mathrm{d}\left[\mathrm{C}_{3} \mathrm{H}_{7} \mathrm{OH}\right]}{\mathrm{d} t}=-k_{2}\left[\mathrm{C}_{3} \mathrm{H}_{7} \mathrm{OH}\right]+k_{-2}\left[\mathrm{C}_{3} \mathrm{H}_{7} \mathrm{OH}^{*}\right]-k_{4}\left[\mathrm{C}_{3} \mathrm{H}_{7} \mathrm{OH}\right][\cdot \mathrm{OH}]
$$

Similarly, the generation rates of $\mathrm{C}_{3} \mathrm{H}_{7} \mathrm{OH}^{*},{ }^{\bullet} \mathrm{OH}, \mathrm{HO}_{2}{ }^{\bullet}$, and $\mathrm{O}_{2}{ }^{\bullet}$ are described as the following Equations (2') $-\left(5^{\prime}\right)$ :

$$
\begin{aligned}
& \frac{\mathrm{d}[\cdot \mathrm{OH}]}{\mathrm{d} t}=2 k_{1}\left[\mathrm{H}_{2} \mathrm{O}_{2}\right]-k_{4}\left[\mathrm{C}_{3} \mathrm{H}_{7} \mathrm{OH}\right][\cdot \mathrm{OH}]-k_{6}\left[\mathrm{H}_{2} \mathrm{O}_{2}\right][\cdot \mathrm{OH}] \\
& -k_{7}\left[\mathrm{H}_{2} \mathrm{O}_{2}\right][\cdot \mathrm{OH}]+k_{8}\left[\mathrm{H}_{2} \mathrm{O}_{2}\right]\left[\mathrm{HO}_{2}^{\cdot}\right]+k_{9}\left[\mathrm{H}_{2} \mathrm{O}_{2}\right]\left[\mathrm{O}_{2}^{\cdot-}\right] \\
& \frac{\mathrm{d}\left[\mathrm{HO}_{2}^{\cdot}\right]}{\mathrm{d} t}=k_{6}\left[\mathrm{H}_{2} \mathrm{O}_{2}\right][\cdot \mathrm{OH}]-k_{8}\left[\mathrm{H}_{2} \mathrm{O}_{2}\right]\left[\mathrm{HO}_{2}^{\cdot}\right] \\
& \frac{\mathrm{d}\left[\mathrm{O}_{2}^{\cdot-}\right]}{\mathrm{d} t}=k_{7}\left[\mathrm{H}_{2} \mathrm{O}_{2}\right][\cdot \mathrm{OH}]-k_{9}\left[\mathrm{H}_{2} \mathrm{O}_{2}\right]\left[\mathrm{O}_{2}^{\cdot-}\right] \\
& \frac{\mathrm{d}\left[\mathrm{C}_{3} \mathrm{H}_{7} \mathrm{OH} *\right]}{\mathrm{d} t}=k_{2}\left[\mathrm{C}_{3} \mathrm{H}_{7} \mathrm{OH}\right]-k_{-2}\left[\mathrm{C}_{3} \mathrm{H}_{7} \mathrm{OH} *\right]-k_{3}\left[\mathrm{C}_{3} \mathrm{H}_{7} \mathrm{OH}^{*}\right]
\end{aligned}
$$

Applying the steady-state approximation to the transitory reactive intermediates:

$$
\frac{\mathrm{d}\left[\bullet^{\cdot \mathrm{OH}}\right]}{\mathrm{d} t}=\frac{\mathrm{d}\left[\mathrm{HO}_{2}{ }^{\bullet}\right]}{\mathrm{d} t}=\frac{\mathrm{d}\left[\mathrm{O}_{2}^{\cdot-}\right]}{\mathrm{d} t}=\frac{\mathrm{d}\left[\mathrm{C}_{3} \mathrm{H}_{7} \mathrm{OH}^{*}\right]}{\mathrm{d} t}=0
$$

The concentration of ${ }^{\bullet} \mathrm{OH}$ and $\mathrm{C}_{3} \mathrm{H}_{7} \mathrm{OH}^{*}$ are, therefore, given by Equations (7') and (8'), respectively:

$$
\begin{aligned}
& {[\cdot \mathrm{OH}]=\frac{2 k_{1}\left[\mathrm{H}_{2} \mathrm{O}_{2}\right]}{k_{4}\left[\mathrm{C}_{3} \mathrm{H}_{7} \mathrm{OH}\right]}} \\
& {\left[\mathrm{C}_{3} \mathrm{H}_{7} \mathrm{OH}^{*}\right]=\frac{k_{2}\left[\mathrm{C}_{3} \mathrm{H}_{7} \mathrm{OH}\right]}{k_{-2}+k_{3}}}
\end{aligned}
$$


Substituting (7') and (8') into Equation (1'), and thus the degradation rate of IPA can be rewritten as follows:

$$
\begin{aligned}
-\frac{\mathrm{d}\left[\mathrm{C}_{3} \mathrm{H}_{7} \mathrm{OH}\right]}{\mathrm{d} t} & =k_{2}\left[\mathrm{C}_{3} \mathrm{H}_{7} \mathrm{OH}\right]+k_{4}\left[\mathrm{C}_{3} \mathrm{H}_{7} \mathrm{OH}\right] \frac{2 k_{1}\left[\mathrm{H}_{2} \mathrm{O}_{2}\right]}{k_{4}\left[\mathrm{C}_{3} \mathrm{H}_{7} \mathrm{OH}\right]}-k_{-2} \times \frac{k_{2}\left[\mathrm{C}_{3} \mathrm{H}_{7} \mathrm{OH}\right]}{k_{-2}+k_{3}} \\
& =2 k_{1}\left[\mathrm{H}_{2} \mathrm{O}_{2}\right]+\left(k_{2}-\frac{k_{2} k_{-2}}{k_{-2}+k_{3}}\right)\left[\mathrm{C}_{3} \mathrm{H}_{7} \mathrm{OH}\right]
\end{aligned}
$$

In the case of $\left[\mathrm{H}_{2} \mathrm{O}_{2}\right]_{\mathrm{i}} \gg\left[\mathrm{C}_{3} \mathrm{H}_{7} \mathrm{OH}\right]_{\mathrm{i}}$, the $2 k_{1}\left[\mathrm{H}_{2} \mathrm{O}_{2}\right]$ term can be assumed to be constant, so Equation (9') will become as:

$-\frac{\mathrm{d}\left[\mathrm{C}_{3} \mathrm{H}_{7} \mathrm{OH}\right]}{\mathrm{d} t}=k_{1}^{\prime}+k_{2}^{\prime}\left[\mathrm{C}_{3} \mathrm{H}_{7} \mathrm{OH}\right]$

where $k_{1}^{\prime}=2 k_{1}\left[\mathrm{H}_{2} \mathrm{O}_{2}\right]$ and $k_{2}^{\prime}=\left(k_{2}-\frac{k_{2} k_{-2}}{k_{-2}+k_{3}}\right)$. 


\section{Text S2. Specific energy consumption (SEC) calculation}

Specific energy consumption is estimated using Equation (10').

$\operatorname{SEC}(\mathrm{kWh} / \mathrm{g})=\frac{\mathrm{P} \times \mathrm{t}}{\left(\mathrm{C}_{\mathrm{i}}-\mathrm{C}_{\mathrm{t}}\right) \times \mathrm{V} \times 10^{-6}}$

where $\mathrm{P}$ represents the power consumption in kilowatt; $\mathrm{t}$ is the reaction time in hours; $\mathrm{C}_{\mathrm{i}}$ and $\mathrm{C}_{\mathrm{t}}$ are the initial IPA concentration and residual IPA concentration determined at the reaction time $\mathrm{t}(\mathrm{mg} / \mathrm{L})$, respectively; and $\mathrm{V}$ is the simulated wastewater volume in milliliters.

Table S1. Detailed calculation of specific energy consumption in different treatment systems

\begin{tabular}{|c|c|c|}
\hline & $\mathrm{TH} / \mathrm{H}_{2} \mathrm{O}_{2}$ system & $\mathbf{M W} / \mathrm{H}_{2} \mathrm{O}_{2}$ system \\
\hline $\mathbf{P}$ & $0.36 \mathrm{~kW}$ (average) & $\begin{array}{l}\mathbf{0 . 5 6} \mathrm{kW} \text { (average) for the first } 13 \text { min of } \\
\text { irradiation then reduced and maintained at } \mathbf{0 . 4 0} \\
\mathrm{kW}\end{array}$ \\
\hline $\mathbf{t}$ & $1.583 \mathrm{~h}\left(95 \min \times \frac{1 \mathrm{~h}}{60 \mathrm{~min}}\right)$ & $0.300 \mathrm{~h}\left(18 \min \times \frac{1 \mathrm{~h}}{60 \min }\right)$ \\
\hline$C_{i}-C_{t}$ & $\begin{array}{c}\mathbf{1 6 3 4 . 8 8} \mathrm{mg}-\mathrm{IPA} / \mathrm{L} \\
(2400 \mathrm{mg}-\mathrm{IPA} / \mathrm{L}-765.12 \mathrm{mg}-\mathrm{IPA} / \mathrm{L})\end{array}$ & $\begin{array}{c}\mathbf{1 6 3 4 . 8 8} \mathrm{mg}-\mathrm{IPA} / \mathrm{L} \\
(2400 \mathrm{mg}-\mathrm{IPA} / \mathrm{L}-765.12 \mathrm{mg}-\mathrm{IPA} / \mathrm{L})\end{array}$ \\
\hline $\mathbf{V}$ & $10 \mathrm{~mL}$ & $10 \mathrm{~mL}$ \\
\hline SEC & $34.865 \mathrm{kWh} / \mathrm{g}$-IPA & $9.460 \mathrm{kWh} / \mathrm{g}-\mathrm{IPA}$ \\
\hline
\end{tabular}


Table S2. Toxicity-weighted of IPA and its metabolites in $\mathrm{MW} / \mathrm{H}_{2} \mathrm{O}_{2}$ system

\begin{tabular}{|c|c|c|c|c|c|}
\hline \multirow[t]{2}{*}{ Compound } & \multirow[t]{2}{*}{ Formula } & \multirow{2}{*}{$\begin{array}{c}\text { Lethal } \\
\text { concentration } 50 \\
\left(\mathbf{L C}_{\mathbf{5 0}}, \mathbf{m M}\right)\end{array}$} & \multirow[t]{2}{*}{ Organism } & \multicolumn{2}{|c|}{$\begin{array}{l}\text { Toxicity-weighted } \\
\text { [compound]/LC }\end{array}$} \\
\hline & & & & Influent & $\begin{array}{l}\text { After } 90 \text { min } \\
\text { irradiation }\end{array}$ \\
\hline Isopropyl alcohol & $\left(\mathrm{CH}_{3}\right)_{3} \mathrm{CHOH}$ & $200.00^{\mathrm{a}}$ & Rat & 0.2 & 0.0010 \\
\hline Acetone & $\mathrm{CH}_{3} \mathrm{COCH}_{3}$ & $356.93^{\mathrm{b}}$ & Rat & 0 & 0.0281 \\
\hline Pyruvic acid & $\mathrm{CH}_{3} \mathrm{COCOOH}$ & $\mathrm{NA}^{\mathrm{c}}$ & - & - & - \\
\hline Glyoxylic acid & $\mathrm{OCHCOOH}$ & $\mathrm{NA}^{\mathrm{d}}$ & - & - & - \\
\hline Oxalic acid & $\mathrm{HOOCCOOH}$ & $\mathrm{NA}^{\mathrm{e}}$ & - & - & - \\
\hline Acetic acid & $\mathrm{CH}_{3} \mathrm{COOH}$ & $266.67^{f}$ & Rat & 0 & 0.0054 \\
\hline Formic acid & $\mathrm{HCOOH}$ & $170.72^{\mathrm{g}}$ & Rat & 0 & 0.0218 \\
\hline \multicolumn{4}{|c|}{ Sum of the toxicity-weighted contaminant concentrations (CTI)* } & 0.2 & 0.0562 \\
\hline
\end{tabular}

NA: not available

a, b, f The National Institute for Occupational Safety and Health (NIOSH)

${ }^{\mathrm{c}}$ Safety data sheet (SDS) issued by Musashino Chemical Laboratory, Ltd.

${ }^{\mathrm{d}}$ Material safety data sheet (MSDS) issued by EMD Millipore Corporation

e SDS issued by Carl Roth Gmbh \& Co. Kg, T113

g SDS issued by Newcomer Supply

*Cytotoxicity index (CTI) is calculated as follows:

$$
\mathrm{CTI}=\sum\left(\frac{[\text { compound }]}{\mathrm{LC}_{50}}\right)
$$




\section{FIGURE}

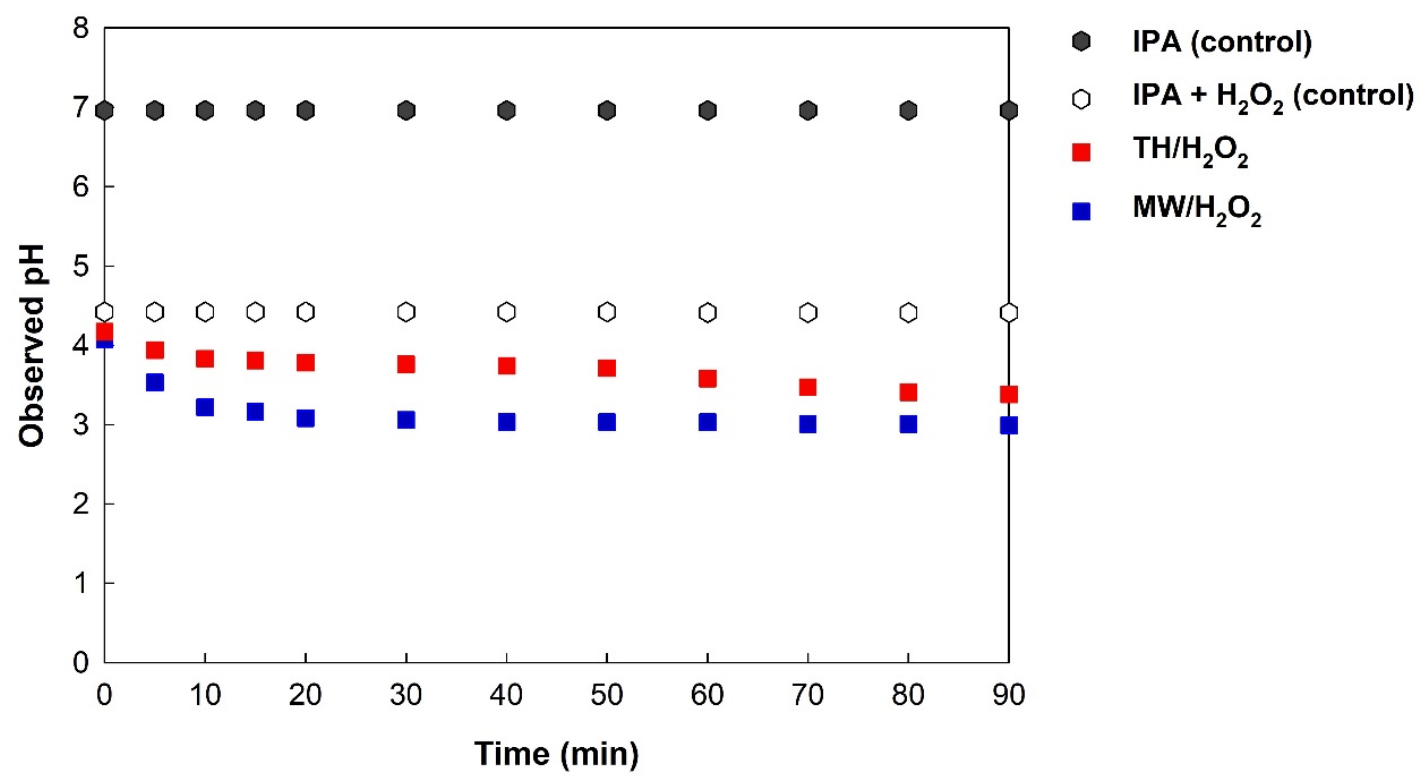

Figure S1. pH observed during the IPA degradation experiments. Operational conditions: $\left[\mathrm{C}_{3} \mathrm{H}_{7} \mathrm{OH}\right]_{\mathrm{i}}=40 \mathrm{mM},\left[\mathrm{H}_{2} \mathrm{O}_{2}\right]_{\mathrm{i}}=0.9 \mathrm{M}, \mathrm{MW}$ power input $=800 \mathrm{~W}$, and pre-heating time $=5$ $\min$.

$\mathrm{MW} / \mathrm{H}_{2} \mathbf{O}_{2}$

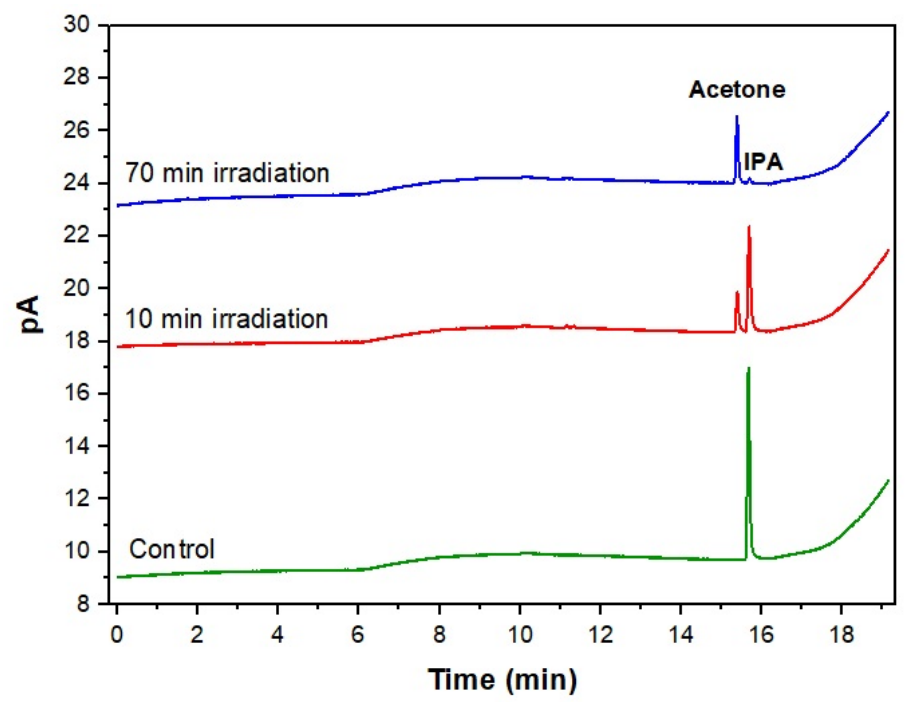

$\mathrm{TH} / \mathrm{H}_{2} \mathrm{O}_{2}$

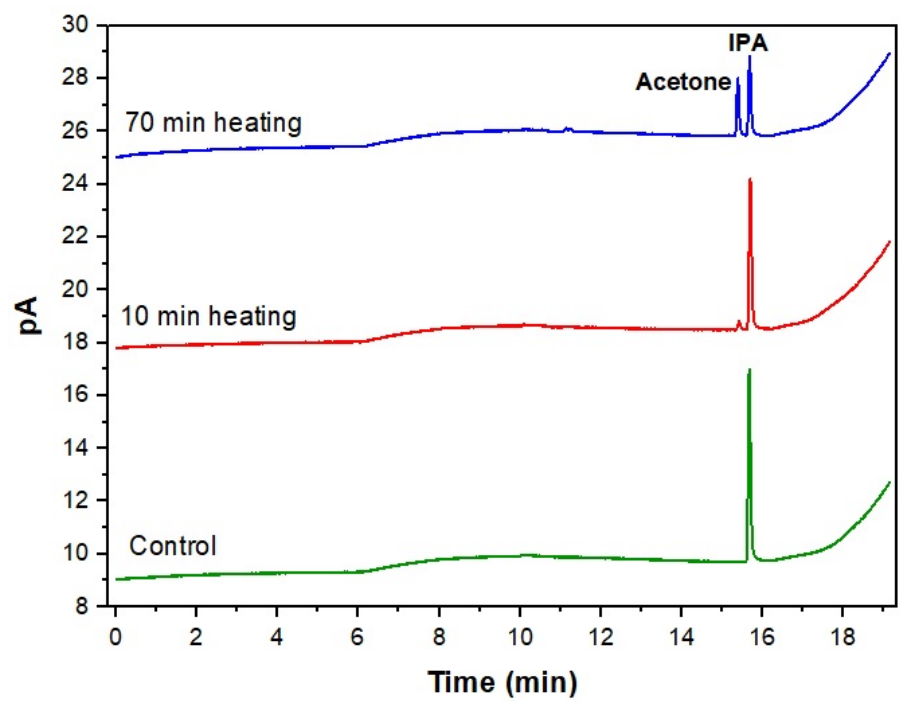

Figure S2. Evolution of GC chromatogram of IPA and its primary degradation product (acetone) under the influence of microwave and thermal treatments. Experimental conditions: $\left[\mathrm{C}_{3} \mathrm{H}_{7} \mathrm{OH}\right]_{\mathrm{i}}=40 \mathrm{mM},\left[\mathrm{H}_{2} \mathrm{O}_{2}\right]_{\mathrm{i}}=0.9$ $\mathrm{M}, \mathrm{MW}$ power input $=800 \mathrm{~W}$, and pre-heating time $=5 \mathrm{~min}$. 
$\mathrm{MW} / \mathrm{H}_{2} \mathbf{O}_{2}$

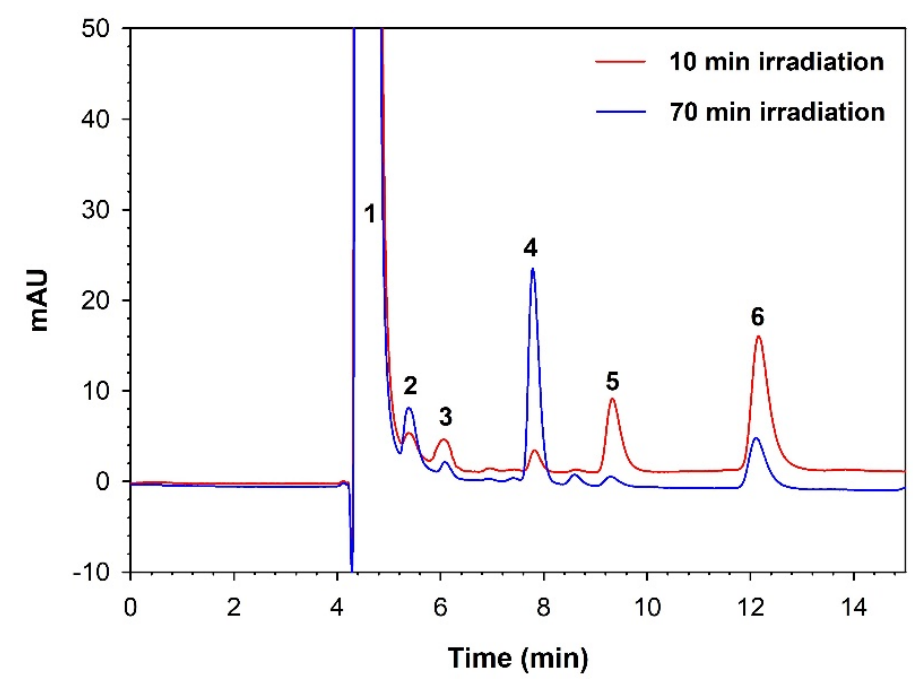

$\mathrm{TH} / \mathrm{H}_{2} \mathrm{O}_{2}$

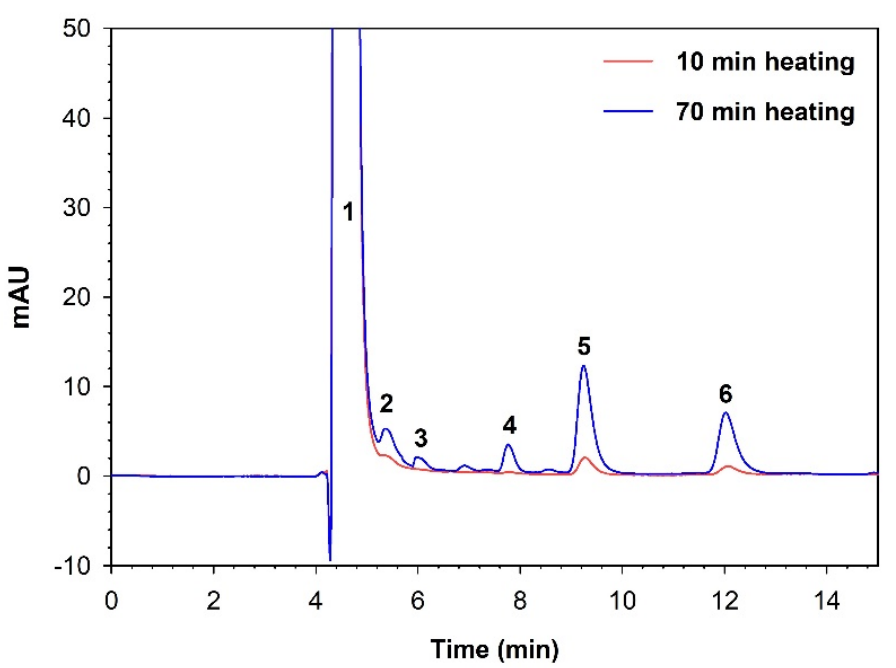

Figure S3. HPLC chromatogram of the generated organic acids in microwave and thermal treatment systems under the detection wavelength of $210 \mathrm{~nm}$, where:

(1) Oxalic acid

(2) Formic acid

(3) Glyoxylic acid

(4) Acetic acid

(5) Unknown

(6) Pyruvic acid

Experimental conditions: $\left[\mathrm{C}_{3} \mathrm{H}_{7} \mathrm{OH}\right]_{\mathrm{i}}=40 \mathrm{mM},\left[\mathrm{H}_{2} \mathrm{O}_{2}\right]_{\mathrm{i}}=0.9 \mathrm{M}, \mathrm{MW}$ power input $=800 \mathrm{~W}$, and pre-heating time $=5$ min. 


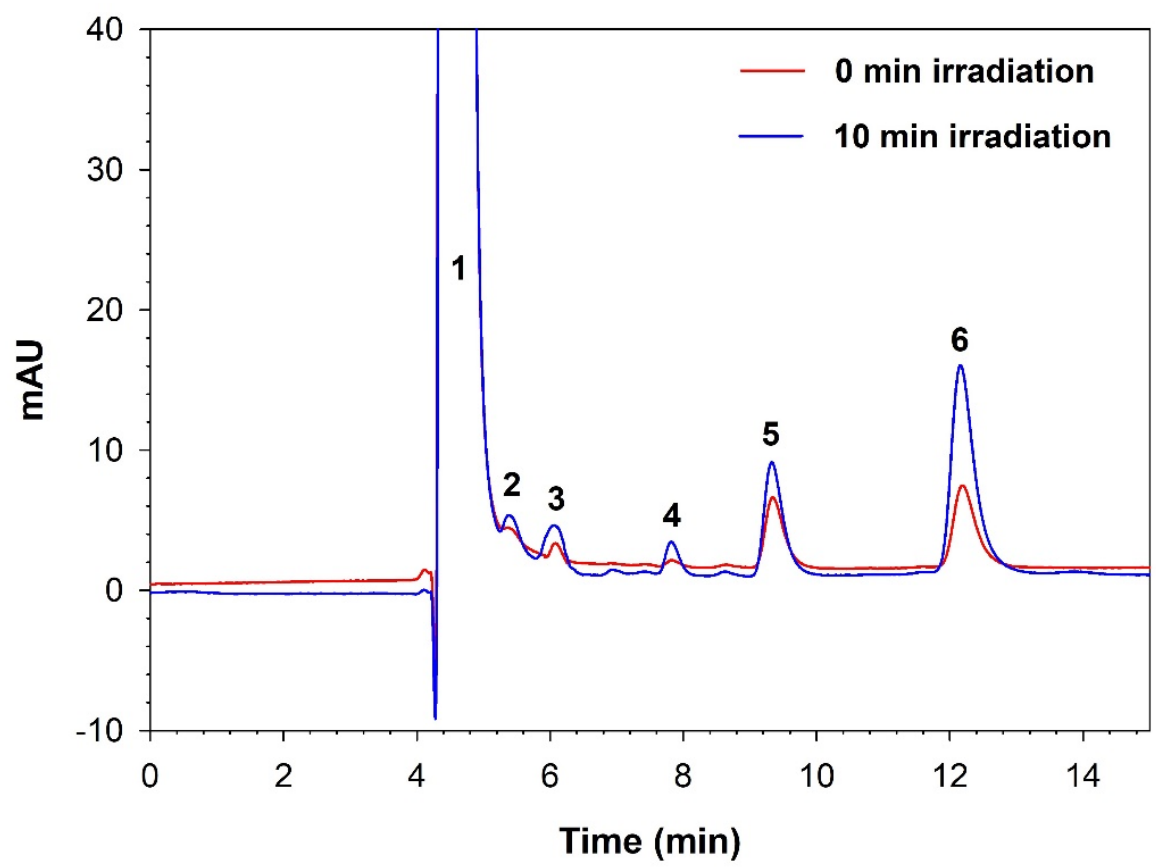

Figure S4. Evolution of HPLC chromatogram of the generated organic acids in microwave treatment system $\left(\mathrm{MW} / \mathrm{H}_{2} \mathrm{O}_{2}\right)$ by the reaction time, where:

- At the reaction time of 0 min, oxalic acid (1), glyoxylic acid (3), and pyruvic acid (6) were detected;

- At the reaction time of $10 \mathrm{~min}$, all intermediates were detected.

Experimental conditions: $\left[\mathrm{C}_{3} \mathrm{H}_{7} \mathrm{OH}\right]_{\mathrm{i}}=40 \mathrm{mM},\left[\mathrm{H}_{2} \mathrm{O}_{2}\right]_{\mathrm{i}}=0.9 \mathrm{M}, \mathrm{MW}$ power input $=800 \mathrm{~W}$, and pre-heating time $=5 \mathrm{~min}$. 


\section{SCHEME}

Scheme S1 - Reaction pathways of the generation and degradation of pyruvic acid

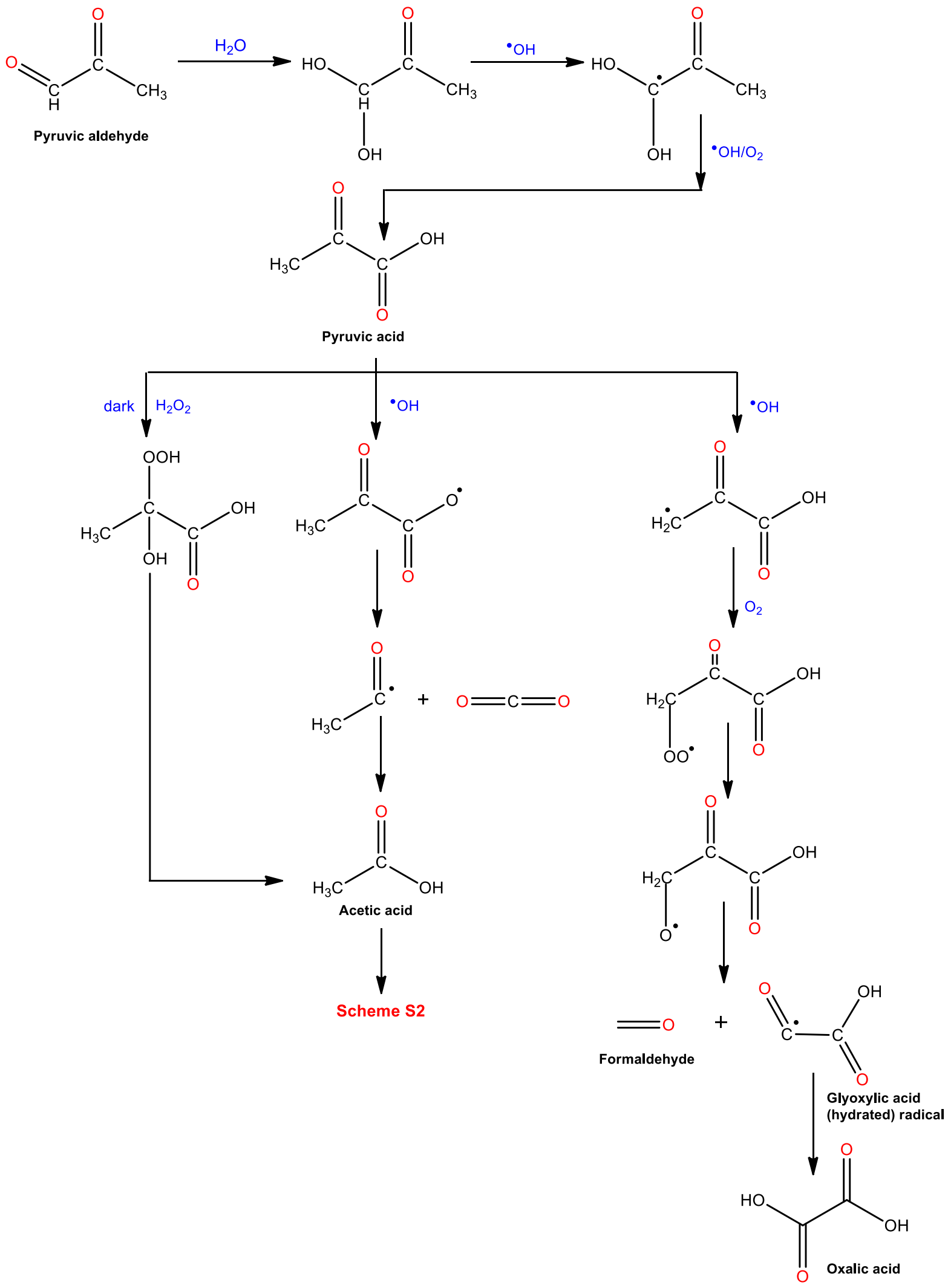


Scheme S2 - Degradation pathways of acetic acid

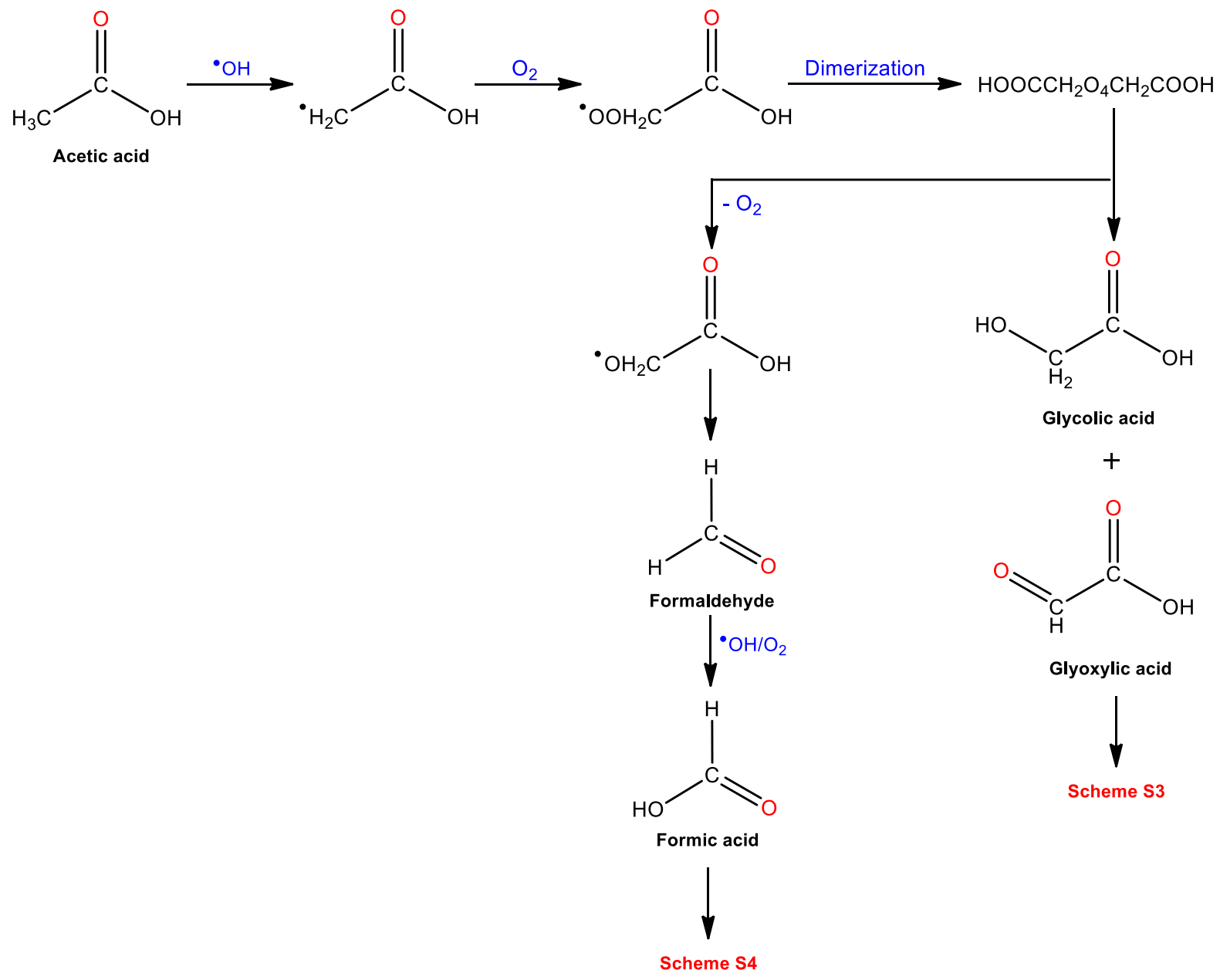


Scheme S3 - Degradation pathways of glycolic acid, glyoxylic acid, and oxalic acid

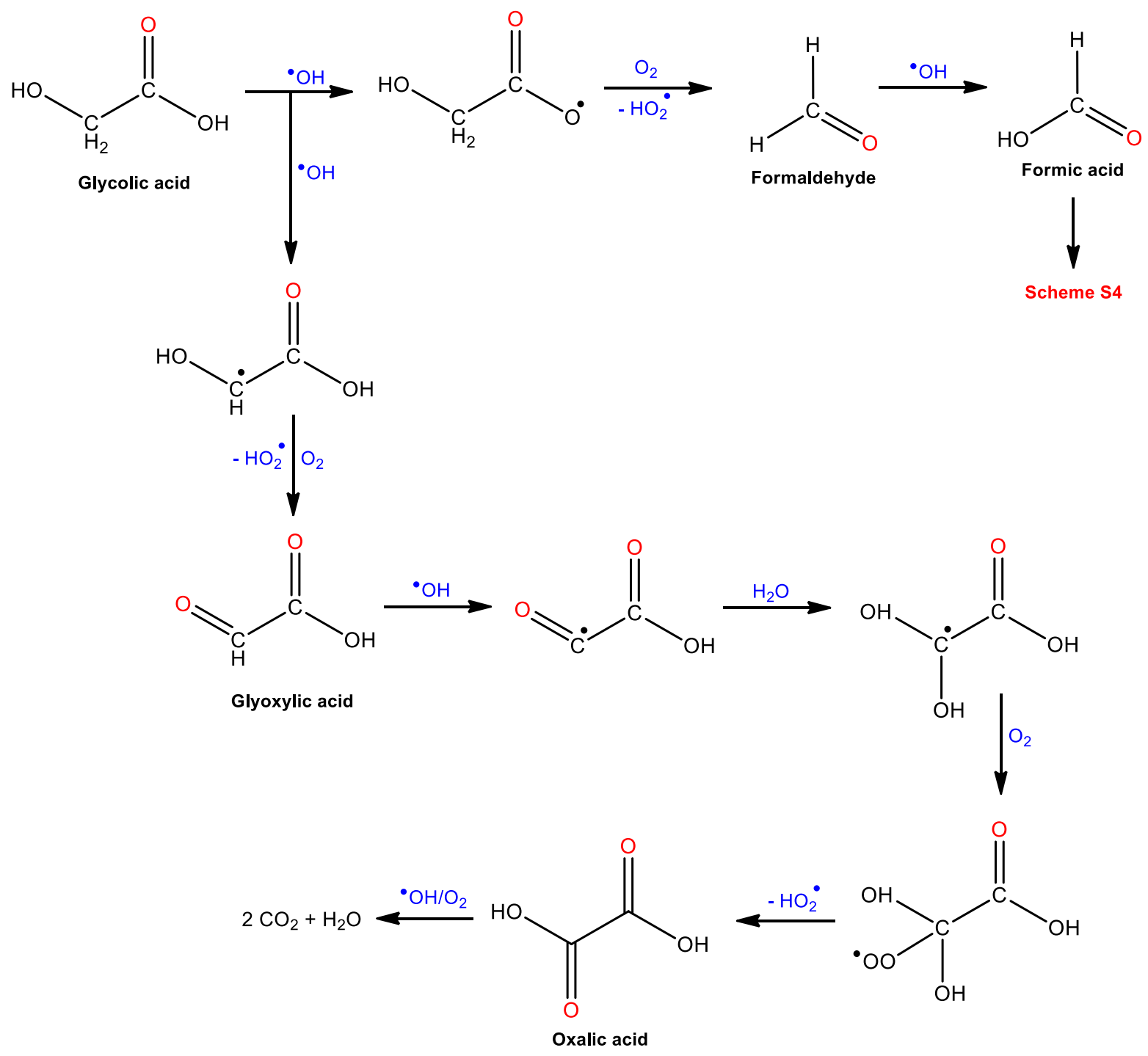

Scheme S4 - Degradation pathways of formic acid

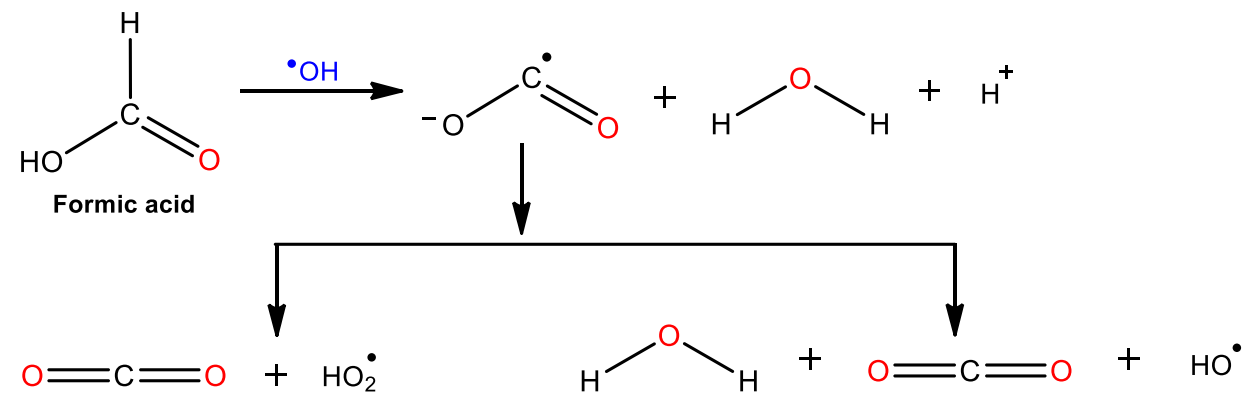

